\title{
Fitness versus Fatness and Insulin Resistance in U.S. Adolescents
}

\author{
Doyle M. Cummings, ${ }^{1}$ Katrina D. DuBose, ${ }^{2}$ Satomi Imai, ${ }^{3}$ and David N. Collier ${ }^{4}$ \\ ${ }^{1}$ Brody School of Medicine, East Carolina University, 600 Moye Boulevard, Greenville, NC 27834, USA \\ ${ }^{2}$ College of Health and Human Performance, East Carolina University, Greenville, NC 27858, USA \\ ${ }^{3}$ Center for Health Services Research and Development, East Carolina University, Greenville, NC 27858, USA \\ ${ }^{4}$ Pediatric Healthy Weight Research and Treatment Center, East Carolina University, Greenville, NC 27858, USA
}

Correspondence should be addressed to Doyle M. Cummings, cummingsd@ecu.edu

Received 27 May 2009; Revised 11 October 2009; Accepted 13 December 2009

Academic Editor: Andrew P. Hills

Copyright (C) 2010 Doyle M. Cummings et al. This is an open access article distributed under the Creative Commons Attribution License, which permits unrestricted use, distribution, and reproduction in any medium, provided the original work is properly cited.

\begin{abstract}
Background. The present study examined the relationship between insulin resistance and both waist circumference and cardiorespiratory fitness in U.S. adolescents. Methods. NHANES assessed a nationally representative sample of U.S. adolescents (12-18 yrs) between 1999-2002. Abdominal adiposity was estimated by waist circumference, overall adiposity by BMI, and cardiorespiratory fitness (maximal oxygen uptake $\left(\mathrm{VO}_{2} \mathrm{max}\right)$ from a treadmill exercise test). Insulin resistance was estimated from fasting insulin and glucose using the homeostatic model assessment method (i.e., HOMA) and was log-transformed. Results. 1078 adolescents were included in the study. Positive correlations existed between lnHOMA and waist circumference $(r=0.59 ; r=0.54)$ for boys and girls, respectively. $\ln \mathrm{HOMA}$ and $\mathrm{VO}_{2}$ max were inversely related in boys $(r=-0.29)$ but not girls $(r=-0.06$. $)$ Gender-specific analyses by BMI category showed that the significant inverse relationship in lnHOMA and $\mathrm{VO}_{2}$ max was primarily present in obese boys. Conclusion. Among adolescents, important gender and BMI differences exist in the relationship between insulin resistance and fitness. While waist circumference and BMI are important predictors in all children, fitness appears especially important in obese boys. These findings may have important implications for gender-specific interventions to prevent adult obesity and diabetes mellitus.
\end{abstract}

\section{Introduction}

Recent studies have identified a growing epidemic of childhood obesity in the United States [1,2] and it has been estimated that one in three US children born in 2000 will develop diabetes mellitus at some point in their lifetime [3]. Hannon et al. [4] have reviewed the pathophysiologic relationship between childhood obesity and the subsequent development of Type 2 diabetes mellitus. The importance of insulin resistance in this process was underscored by Bacha et al. [5] who demonstrated that, in obese adolescents of similar body mass index (BMI), the relative magnitude of insulin resistance at any given BMI value contributed substantially to the risk for metabolic comorbidities and diabetes mellitus.

In addition to obesity levels, cardioresipratory fitness may influence insulin resistance; however, to what degree is still unclear. Data in adults suggests that abdominal adiposity is a stronger predictor of insulin resistance than fitness [6].
This relationship in children has been the subject of multiple investigations [7-11] with varying results. Works by Ball et al. [9], and Krekoukia et al. [12] in children describe the relatively greater importance of central adiposity/waist circumference as a determinant of insulin resistance. In contrast, Allen et al. [7] and Kasa-Vubu et al. [13] suggest that cardiorespiratory fitness is of greater importance as a determinant of insulin resistance in children and young adults. Imperatore et al. [14] describe the importance of cardiorespiratory fitness to insulin sensitivity, but did not study the relative contribution of abdominal adiposity. While, Lee et al. [15] first described the distribution of insulin resistance in adolescents using the homeostatic model assessment (HOMA), they did not examine the relative contributions of either abdominal adiposity or cardiorespiratory fitness. Rizzo et al. [16] and Lee et al. [11] reported that the association between cardiorespiratory fitness and insulin sensitivity in children is mediated by the degree of 
adiposity. Further, Eisenmann et al. [10] found that, within a BMI category, increased cardiorespiratory fitness improved insulin sensitivity in children.

Data reported by Pate et al. [17] from NHANES on 12-19 years old adolescents suggests that cardiorespiratory fitness is higher in boys than in girls and is reduced in overweight adolescents; however, the authors did not examine the relationship to insulin resistance. Given the inconclusive results, the purpose of this study was to examine the relationship of waist circumference and cardiorespiratory fitness to insulin resistance by weight category, race, gender, and age in a large nationally representative sample of US adolescents, aged $12-18$ years.

\section{Methods}

The National Health and Nutrition Examination Survey (NHANES) is conducted annually by the National Center for Health Statistics of the Centers for Disease Control and Prevention (CDC) and provides data on a variety of health and nutritional parameters for US citizens. NHANES uses complex probability sampling with a cluster sample design. The 1999-2002 NHANES data was chosen for the present study because adolescents aged 12-19 years old were oversampled in these years to provide more precise population estimates. The NHANES protocol was approved by the CDC's institutional review board and written informed consent, from legal guardians, and minor assent was obtained.

The present study included all children aged 12-18 years old who participated in the fasting subsample of the NHANES 1999-2002 survey and who had available cardiorespiratory fitness data. Data was analyzed in 2008. Those excluded from the study were those who did not have a fasting blood sample or those who were excluded from exercise treadmill testing because of cardiovascular or pulmonary conditions, morbid obesity, pregnancy, amputation or disability, or other conditions, or medication use that might pose a risk.

BMI was calculated from height and weight measured by trained technicians using methods described by the NHANES manual for anthropometrics [18-20]. Using the CDC growth charts for US children, BMI values were then used to categorize each adolescent as normal weight (5th-84th percentile), overweight (85th-94th percentile), or obese ( $\geq 95$ th percentile) [21]. Waist circumference, a measure of abdominal adiposity, was measured to the nearest $0.1 \mathrm{~cm}$ at the highest point of the iliac crest. The technicians were trained on the proper procedures for measuring waist circumference and reliability checks were conducted throughout the data collection process [20]. Waist circumference was chosen as a measure of abdominal adiposity in this study because of its availability in NHANES databases, because it can be easily and reliably measured in primary care practices, and because it is associated with cardiovascular risk [22-24].

Cardiorespiratory fitness was measured using a submaximal exercise treadmill test using a protocol specific to the individual's predicted fitness level and designed to achieve $80 \%$ of the age-predicted maximal heart rate [25]. The technicians were trained on the proper procedures for administering the aerobic fitness test and reliability checks were conducted throughout the data collection process by experienced personnel [25]. Estimated maximal oxygen uptake $\left(\mathrm{VO}_{2} \max \right)$ was determined by extrapolation of data from the heart rate response following the two main stages of the treadmill exercise test to an expected age-specific heart rate standard as specified by NHANES.

Venous blood was obtained following at least an 8hour fast and was analyzed for glucose by the hexokinase technique and for insulin by radioimmunoassay technique as described in the NHANES methods [26]. The homeostatic model assessment (HOMA) was used to estimate insulin resistance, based on the work of Keskin et al. [27] who found it to have acceptable sensitivity and specificity in diagnosing insulin resistance in children. HOMA was calculated from fasting glucose and insulin as follows: the product of the fasting value of glucose (expressed as $\mathrm{mg} / \mathrm{dL}$ ) and insulin (expressed as $\mu \mathrm{U} / \mathrm{mL}$ ) is divided by 405 . The resulting value was subsequently log transformed (nHOMA) to normalize the distribution.

For statistical analysis, univariate comparisons utilized Pearson correlation coefficients for continuous variables (i.e., age, BMI, waist circumference, $\mathrm{VO}_{2} \max$, and $\ln \mathrm{HOMA}$ ) using SPSS (Version 15, Statistical Package for the Social Sciences, Chicago; IL). T-tests and analysis of variance with Bonferoni correction were used for multiple within gender comparisons of means by tertiles (high fitness for boys, girls: $\mathrm{VO}_{2} \max \geq 50.52,41.22$, resp.) (moderate fitness for boys, girls: $\mathrm{VO}_{2} \max \geq 42.99,35.81 ; \mathrm{VO}_{2} \max <50.52,41.22$, resp.) and (low fitness for boys, girls; $\mathrm{VO}_{2} \max \geq 42.99$, 35.81, resp.) of cardiorespiratory fitness using SAS-callable SUDAAN (version 9, SAS, Cary, NC).

Multivariate analysis involved the construction of several linear regression models. These models examined the relative association of waist circumference, $\mathrm{BMI}$, and $\mathrm{VO}_{2}$ max with InHOMA by gender, while controlling for the effects of age (in months), and race (Caucasian versus non-Caucasian). In the final regression model, waist circumference and $\mathrm{VO}_{2} \max$ were compared directly in all adolescents and in those with a normal BMI versus those over the 85th percentile, and BMI was excluded from the model because of the potential interaction of BMI and waist circumference.

All analyses utilized NHANES weights to accommodate for the complex sampling design of NHANES. The weights account for the geographic probability sampling methods, the oversampling of specific groups such as minorities and adolescents, and are designed to insure the representativeness of the weighted findings to that of all adolescents in the U.S. Statistical significance was set at alpha $<0.05$.

\section{Results}

A total of 1,078 adolescents from the NHANES database aged 12-18 years with complete data were included in the present analysis and their demographic characteristics are shown in Table 1. Forty-six percent of the participants were girls and 38\% were non-Caucasian (includes 14\% African 
TABLE 1: Characteristics of the study sample by gender.

\begin{tabular}{lccr}
\hline Parameter & Boys $(n=573)($ mean \pm SEM $)$ & Girls $(n=505)($ mean \pm SEM $)$ & All $(N=1078)(\mathrm{mean} \pm$ SEM $)$ \\
\hline Age $(\mathrm{yr})$ & $15.14 \pm 0.07$ & $14.92 \pm 0.13$ & $15.03 \pm 0.08$ \\
$\mathrm{BMI}\left(\mathrm{kg} \cdot \mathrm{m}^{-2}\right)$ & $22.72 \pm 0.30$ & $22.58 \pm 0.33$ & $22.65 \pm 0.24$ \\
Waist circumference $(\mathrm{cm})$ & $79.99 \pm 0.79$ & $77.95 \pm 0.91$ & $78.99 \pm 0.68$ \\
$\mathrm{VO}_{2} \mathrm{max}\left(\mathrm{mL} \cdot \mathrm{kg}^{-1} \cdot \mathrm{min}^{-1}\right)$ & $47.4 \pm 0.64^{*}$ & $39.59 \pm 0.44$ & $43.57 \pm 0.52$ \\
$\mathrm{HOMA}$ & $2.83 \pm 0.11$ & $2.92 \pm 0.11$ & $2.87 \pm 0.07$ \\
Obesity status $(\%)$ & & 73 & 70 \\
$\quad$ Normal weight & 67 & 27 & 30 \\
Overweight/obese & 33 & & \\
\hline
\end{tabular}

BMI: body mass index; ${ }^{*} P<.01$ boys versus girls.

TABLE 2: Pearson product moment correlation coefficients between waist circumference, $\mathrm{VO}_{2}$ max, $\mathrm{BMI}$, and $\ln \mathrm{HOMA}$ by gender.

\begin{tabular}{|c|c|c|c|}
\hline \multirow[b]{2}{*}{ Parameter } & \multicolumn{3}{|c|}{ LnHOMA } \\
\hline & Boys $(n=573)$ & Girls $(n=505)$ & All $(N=1078)$ \\
\hline Waist circumference & $0.59 *$ & $0.54^{*}$ & $0.56^{*}$ \\
\hline $\mathrm{VO}_{2} \max$ & $-0.29^{*}$ & -0.06 & $-0.20^{*}$ \\
\hline BMI & $0.57 *$ & $0.56^{*}$ & $0.56^{*}$ \\
\hline
\end{tabular}

BMI: body mass index; ${ }^{*} P<.01$.

American, 11\% Mexican American, and 13\% other races). The majority of participants were normal weight and $36 \%$ were either overweight or obese. The distribution of obesity status was similar between boys and girls. Estimated $\mathrm{VO}_{2} \max$ was significantly higher in boys than girls $(P<.01)$; however, there was no significant difference in waist circumference by gender. The mean age for the participants in the normal, overweight, and obese groups was similar; however, mean HOMA was significantly higher in the overweight (3.0 versus $2.3, P<.001)$ and obese (5.4 versus $2.3, P$ $<.001)$ groups than in normal weight adolescents. Likewise, mean waist circumference was significantly higher in the overweight ( 85.4 versus $73.5 \mathrm{~cm}, P<.001)$ and obese (100.7 versus $73.5 \mathrm{~cm}, P<.001)$ groups than in normal weight adolescents. In contrast, estimated $\mathrm{VO}_{2}$ max was higher in the normal weight group $\left(44.5 \mathrm{~mL} \cdot \mathrm{kg}^{-1} \cdot \mathrm{min}^{-1}\right)$ compared to the overweight $\left(41.5 \mathrm{~mL} \cdot \mathrm{kg}^{-1} \cdot \mathrm{min}^{-1}, P<.05\right)$ and obese $\left(41.3 \mathrm{~mL} \cdot \mathrm{kg}^{-1} \cdot \mathrm{min}^{-1}, P<.01\right)$ groups.

Table 2 shows the correlation coefficients between lnHOMA, $\mathrm{VO}_{2} \max$, waist circumference, and $\mathrm{BMI}$ for the entire sample and by gender. For the total sample, a positive and significant relationship existed between InHOMA and both waist circumference and BMI. There was a modest inverse relationship between InHOMA and estimated $\mathrm{VO}_{2}$ max.

When the correlations were separated by gender, the moderate and significantly positive correlations were still observed between InHOMA and both waist circumference and BMI for both boys and girls. Further, a modest yet significant inverse correlation was present between InHOMA and estimated $\mathrm{VO}_{2}$ max for boys, but not for girls. To clarify the role of estimated $\mathrm{VO}_{2}$ max within gender, we separated boys and girls into tertiles based on their estimated $\mathrm{VO}_{2} \max$ and found a significant pattern of differences in HOMA in boys by fitness level (see Figure 1). By contrast, mean
HOMA values in girls by fitness level did not show a consistent pattern. Interestingly, when we compared normal weight low fitness boys and girls to overweight/obese high fitness boys and girls a difference was only observed among the girls. A subanalysis examined this relationship with $\mathrm{VO}_{2}$ max corrected by lean body mass. Lean body mass was determined by DEXA and was available on 536 adolescents. After correcting for lean body mass, mean HOMA values were similar across fitness and obesity categories for both boys and girls (data not shown). Therefore, among boys the differences observed in HOMA values by fitness were no longer present.

The initial regression analysis for $\ln \mathrm{HOMA}, \mathrm{BMI}$, waist circumference, and estimated $\mathrm{VO}_{2}$ max for the entire sample and by gender, controlling for race and age is presented in Table 3. For all children, waist circumference, BMI, and estimated $\mathrm{VO}_{2}$ max were significant independent predictors of lnHOMA after adjusting for race and age. Genderspecific analyses showed that BMI remained a significant independent predictor of InHOMA in both boys and girls. However, waist circumference and estimated $\mathrm{VO}_{2}$ max were both significant independent predictors of InHOMA in boys, but not girls. In the final model comparing only waist circumference and estimated $\mathrm{VO}_{2} \max$ without BMI (see Table 3), waist circumference remained a significant independent predictor for both boys and girls while estimated $\mathrm{VO}_{2} \max$ was a significant independent predictor for only boys and not girls. To clarify this further, separate genderspecific regression models were run by BMI group (normal versus $>85$ th percentile), and demonstrated that, among boys, waist circumference was a significant independent predictor of lnHOMA in both BMI groups while cardiorespiratory fitness was a significant independent predictor of InHOMA only in the group above the 85th percentile of BMI for age and gender (data not shown). Among girls, waist circumference was a significant independent predictor of InHOMA in both BMI groups while cardiorespiratory fitness was not a significant independent predictor of InHOMA in either BMI group (data not shown).

\section{Discussion}

The purpose of this study was to examine the relationship of insulin resistance and waist circumference and cardiorespiratory fitness in a large nationally representative sample of 


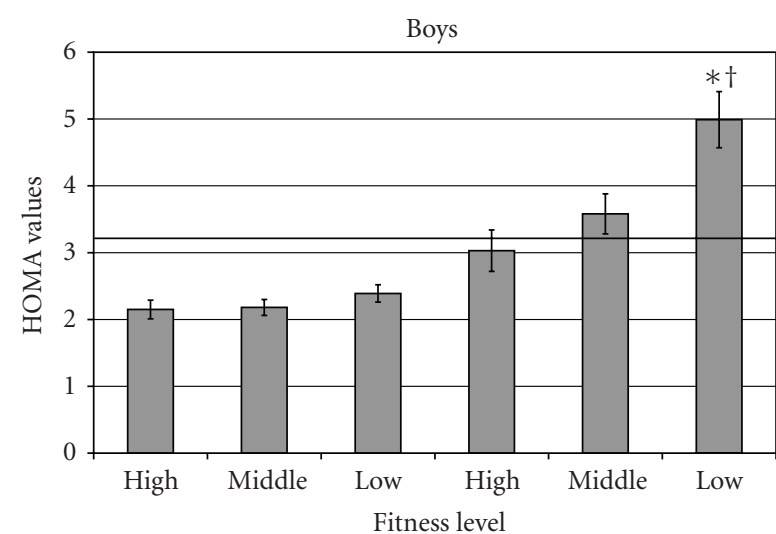

Normal weight

Overweight/obese

* = high versus low fitness (within obesity group), $P<.01$;

$\dagger=$ middle versus low fitness (within obesity group), $P<.01$

High fitness $=\mathrm{VO}_{2} \max \geqslant 50.52 \mathrm{~mL} / \mathrm{kg} / \mathrm{min}^{-1}$

Middle fitness $=\mathrm{VO}_{2} \max \geqslant 42.99<50.52 \mathrm{~mL} / \mathrm{kg} / \mathrm{min}^{-1}$

Low fitness $=\mathrm{VO}_{2} \mathrm{max}<42.99 \mathrm{~mL} / \mathrm{kg} / \mathrm{min}^{-1}$

3.16 = cut-off value for high HOMA values (i.e., insulin resistance)

(Keskin et al., 2005)

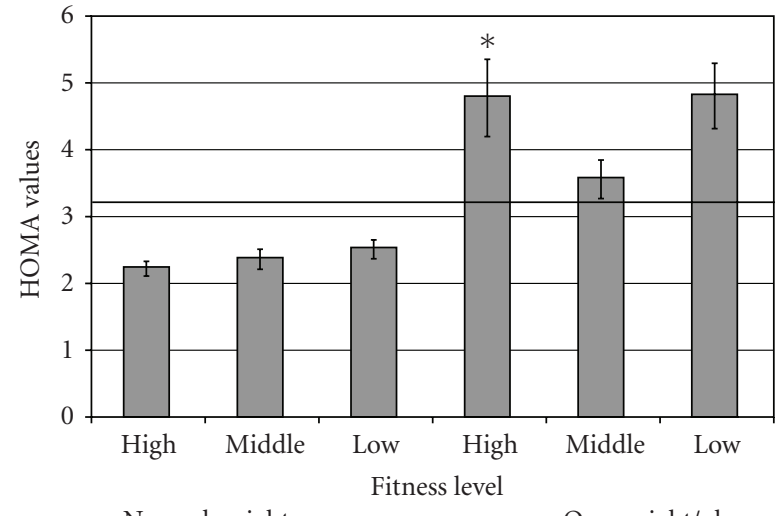

Normal weight

* = Normal weight low fitness versus overweight/obese high, $P<.007$;

High fitness $=\mathrm{VO}_{2} \max \geqslant 41.22 \mathrm{~mL} / \mathrm{kg} / \mathrm{min}^{-1}$

Middle fitness $=\mathrm{VO}_{2} \max \geqslant 35.81<41.22 \mathrm{~mL} / \mathrm{kg} / \mathrm{min}^{-1}$

Low fitness $=\mathrm{VO}_{2} \max <35.81 \mathrm{~mL} / \mathrm{kg} / \mathrm{min}^{-1}$

(b)

FIGURE 1: The relationship between aerobic fitness, obesity level and HOMA levels in boys and girls.

TABLE 3: Regression analysis of the relationship between waist circumference, $\mathrm{VO}_{2} \mathrm{max}, \mathrm{BMI}$, and lnHOMA by gender, while controlling for age and race.

\begin{tabular}{lccr}
\hline \multicolumn{3}{c}{ LnHOMA Beta and 95\% CI } \\
\hline Initial model & Boys $(n=573) R^{2}=0.37$ & Girls $(n=504) R^{2}=0.33$ & All $(N=1077) R^{2}=0.35$ \\
\hline Waist circumference & $0.013(0.0016-0.024)$ & $0.013(-0.001-+0.009)$ & $0.011(0.003-0.019)$ \\
$\mathrm{VO}_{2}$ max & $-0.007(-0.0014--0.001)$ & $-0.0003(-0.007-+0.006)$ & $-0.006(-0.008---0.003)$ \\
$\mathrm{BMI}$ & $0.013(0.0019-0.06)$ & $0.032(0.0095-0.054)$ & $0.032(0.012-0.053)$ \\
Final model & Boys $(n=573) R^{2}=0.37$ & Girls $(n=504) R^{2}=0.31$ & All $(N=1077) R^{2}=0.33$ \\
\hline Waist circumference & $0.023(0.018-0.028)$ & $0.022(0.017-0.027)$ & $-0.006(-0.009---0.003)$ \\
$\mathrm{VO}_{2}$ max & $-0.008(-0.014---0.0015)$ & $0.00(-0.0065-+0.0065)$ & $-0.006(-0.009---0.003)$ \\
\hline
\end{tabular}

BMI: body mass index.

children. Univariate analysis showed that increasing abdominal adiposity, as measured by waist circumference, was associated with increasing insulin resistance, as measured by HOMA. Similar results have been demonstrated by other investigators using various markers for insulin resistance and body composition [7, 12, 28, 29]. After controlling for race and age, increased abdominal adiposity was associated with insulin resistance for the entire sample and in boys and girls. Interestingly, others have reported a relationship between insulin resistance and total percent body fat in boys, but not girls [7]. A possible reason for the difference in results between the current study and Allen et al. [7] is due to participant and methodological differences. In the Allen et al. [7] study, participants were younger (Mean age $=12.9$ years) and were all obese $\left(\mathrm{BMI} \geq 30 \mathrm{~kg} \cdot \mathrm{m}^{-2}\right)$. In contrast, the NHANES sample used in the current study consisted of participants averaging 14 years old and the majority of participants were normal weight. Further, Allen et al. [7] measured percent body fat; whereas the current study was interested in abdominal adiposity as measured via waist circumference.

Abdominal adiposity is not the only concern; overall BMI may also be a factor in insulin resistance. Univariate analysis showed that BMI was related to lnHOMA in the total sample and within each gender. Further, after controlling for race and age, BMI remained as an independent predictor of InHOMA in both genders. Others have reported that HOMA scores and other insulin resistance measures increase as BMI levels increase $[10,12,28]$. The current study also showed that increasing levels of cardiorespiratory fitness are associated with lower levels of insulin resistance for all children. A study by Krekoukia et al. [12] showed that cardiorespiratory fitness was not a significant predictor of insulin resistance. One possible reason for differing results is that Krekoukia et al. [12] measured cardiorespiratory fitness using a cycle ergometer and the age of the participants 
was 9-11 years old, whereas, the current study used a treadmill protocol and the participants were 12-18 years old. The cycle ergometer could have produced localized muscle fatigue earlier than the treadmill, or the lower physiological development of the participants in the Krekoukia et al. [12] study could have limited performance of the exercise test.

When separated by fitness level, significant differences in insulin resistance were observed in boys, but not in girls. Interestingly, when we compared HOMA levels across fitness levels, overweight/obese high fit girls had higher HOMA values compared to normal weight low fit girls. A similar finding was not observed among the boys; the HOMA levels of these two groups were similar in the boys. These results suggest that fitness is an important factor in boys more so than girls. However, in a subanalysis, after adjusting aerobic fitness level by lean body mass, there were no differences in HOMA values within the normal and overweight/obese groups. The lack of difference in HOMA values could be due to similar lean body mass levels among boys within the normal (high fitness $=47.9 \pm 2.5 \mathrm{~kg}$; middle fitness $=46.1$ $\pm 0.9 \mathrm{~kg}$; low fitness $=45.5 \pm 1.3 \mathrm{~kg}$ ) and overweight $/$ obese (high fitness $=57.8 \pm 2.8 \mathrm{~kg}$; middle fitness $=58.1 \pm 3.6 \mathrm{~kg}$; low fitness $=52.0 \pm 1.7 \mathrm{~kg}$ ) groups. To the best of our knowledge other studies have not examined the relationship of fitness and fatness with HOMA by gender in this manner.

In the final regression model, after controlling for race and age, the relationship between insulin resistance and $\mathrm{VO}_{2}$ max was maintained in all adolescents and among boys suggesting a greater relative importance of cardiorespiratory fitness as a predictor of insulin resistance in boys. In particular, this relationship remained significant only in those boys over the 85th percentile of BMI for age and gender, a group at higher risk for adult obesity and diabetes mellitus. This is an important finding and suggests that greater cardiorespiratory fitness in overweight boys leads to biological changes in insulin and glucose metabolism that may be protective with respect to the risk of Type 2 diabetes mellitus. Indeed, Shaibi et al. [30] have shown that exercise in boys can lead to marked improvement in insulin sensitivity without changes in body composition.

By contrast, in girls no relationship between estimated $\mathrm{VO}_{2}$ max and InHOMA was observed, regardless of BMI group, suggesting that cardiorespiratory fitness is a less important predictor in females. These findings support the gender differences observed in a study by Allen et al. [7]. They found that in boys, fitness levels had a stronger relationship with insulin resistance than percent body fat. Whereas, in girls, no relationship between either percent body fat and $\mathrm{HOMA}$ or $\mathrm{VO}_{2}$ max adjusted for lean body mass was observed [7]. Our findings are similar to Allen et al. [7] for the relationship between $\mathrm{VO}_{2}$ max and lnHOMA among girls even though they used a different method to estimate percent body fat and a younger, smaller non-populationbased sample.

The observed gender differences in the current study could be due to the differences in cardiorespiratory fitness [17]. Differences in physical activity participation by the genders might explain why boys have higher cardiorespiratory fitness levels than girls. Girl's physical activity participation, especially vigorous-intensity physical activity, declines as they age more than boys [31]. According to a recent paper that measured physical activity levels objectively, $10 \%-11.9 \%$ of boys from 12 to 19 years old are meeting current physical activity guidelines but only $5.6 \%-7.6 \%$ of girls from 12 to 19 years old are meeting physical activity guidelines [31]. Differences in the relationship between cardiorespiratory fitness and insulin resistance may also relate to the complex and differing physiologic and psychosocial effects of puberty in boys and girls. Girls often have a decline in $\mathrm{VO}_{2} \max$ in late puberty in association with weight gain while boys frequently have an increase in cardiovascular fitness in late puberty [32].

We have found that fitness level $\left(\mathrm{VO}_{2} \max \right)$ is an independent predictor of insulin resistance in boys. Even among boys who are overweight or obese those with the highest fitness levels do not meet criteria for insulin resistance (HOMA > 3.26) while overweight and obese boys in the middle and low tertiles for fitness are insulin resistant (HOMA levels of about 3.5 and 5.0, resp.). In contrast, overweight and obese girls are, on average, all insulin resistant, irrespective of their fitness tertile. Girls in this study had lower fitness levels overall than boys $\left(39.59 \pm 0.44 \mathrm{~mL} \cdot \mathrm{kg}^{-1} \cdot \mathrm{min}^{-1}\right.$ versus $47.4 \pm 0.64 \mathrm{~mL} \cdot \mathrm{kg}^{-1} \cdot \mathrm{min}^{-1}$, resp.). Another reason, other than lower physical activity levels, for the lower fitness levels in girls is due to less lean body mass (muscle mass) than boys. Lean body mass in girls ranged from $34.5 \mathrm{~kg}$ to $44.2 \mathrm{~kg}$, whereas in boys it was between $45.5 \mathrm{~kg}$ and $58.1 \mathrm{~kg}$. Therefore, differences may be due, in large part, to the higher percentage lean body mass (muscle mass) found in boys than in girls [33]. Even the average $\mathrm{VO}_{2}$ max for the highest fitness tertile of overweight and obese girls was lower than that of the lowest fitness tertile for overweight and obese boys (41.22 $\mathrm{mL} \cdot \mathrm{kg}^{-1} \cdot \mathrm{min}^{-1}$ versus $42.99 \mathrm{~mL} \cdot \mathrm{kg}^{-1} \cdot \mathrm{min}^{-1}$, resp.). This observation suggests that fitness levels in overweight and obese girls, irrespective of their fitness tertile, fall below a threshold that protects against insulin resistance in the face of obesity. Hence all overweight and obese girls might benefit from interventions designed not only to reduce abdominal adiposity/waist circumference and BMI (independent predictors of IR) but to increase fitness above this theoretical threshold. In contrast, among overweight and obese boys significant insulin resistance was only seen at the lowest fitness level. Consequently, the relative value of interventions to improve fitness in order to promote insulin sensitivity in most overweight and obese boys requires additional investigation to determine which boys may derive specific benefit in terms of insulin sensitivity.

This study has some limitations; first, this was a crosssectional study design. Given this design it is difficult to identify which factor developed first, insulin resistance or obesity and thus causality cannot be determined. These data are only applicable to children with 12 years or older, as blood samples were not obtained from younger children. Further, for this age group, a clinical standard for measuring insulin resistance is lacking. While there are many different methods, HOMA was used in this study to estimate insulin resistance. HOMA has shown to be a valid and reliable method for quantifying insulin resistance in adolescents $[27,34,35]$. In addition, Tanner staging was not assessed in the NHANES 
testing, so a good measure of sexual development is lacking. Therefore, adolescents of similar chronological age may have been at different stages of puberty, resulting in variability in measures of insulin resistance. Although not as accurate as measures such as dual energy absorptiometry, we measured body fatness by measures commonly used in the clinical setting (i.e., BMI and waist circumference) to facilitate broader applicability. Although a structured protocol and trained measurers were utilized, there is limited information about the exact procedures for confirming the reliability of anthropometric measures. Finally, cardiorespiratory fitness was estimated based on a submaximal treadmill test. While a maximal cardiorespiratory fitness test was not conducted, this is the largest number of adolescents in the US that have completed aerobic fitness testing.

\section{Conclusion}

Among adolescents, important gender and weight category differences exist in the relationship between insulin resistance and cardiorespiratory fitness. While waist circumference and BMI are important predictors of insulin resistance in all children, fitness may be more important in boys, especially overweight boys, than girls. Our results suggest that the determination of cardiorespiratory fitness in boys, using a safe, noninvasive submaximal exercise treadmill test may provide additional clinically relevant information pertaining to insulin resistance. Longitudinal studies are needed to determine if low cardiorespiratory fitness and associated insulin resistance, as shown here, are predictive of higher risks for developing Type 2 diabetes. If so, early determination of cardiorespiratory fitness could help guide not only who is in most need of intervention but also the nature of the interventions offered.

\section{Acknowledgment}

This project was supported in part by a grant from the US Health Resources and Services Administration (D54HP03396); the funding agency had no input into the study design, collection, analysis, or interpretation of data, the writing of the report, or the decision to submit for publication.

\section{References}

[1] C. L. Ogden, M. D. Carroll, L. R. Curtin, M. A. McDowell, C. J. Tabak, and K. M. Flegal, "Prevalence of overweight and obesity in the United States, 1999-2004," Journal of the American Medical Association, vol. 295, no. 13, pp. 1549-1555, 2006.

[2] A. A. Hedley, C. L. Ogden, C. L. Johnson, M. D. Carroll, L. R. Curtin, and K. M. Flegal, "Prevalence of overweight and obesity among US children, adolescents, and adults, 19992002," Journal of the American Medical Association, vol. 291, no. 23, pp. 2847-2850, 2004.

[3] K. M. V. Narayan, J. P. Boyle, T. J. Thompson, S. W. Sorensen, and D. F. Williamson, "Lifetime risk for diabetes mellitus in the United States," Journal of the American Medical Association, vol. 290, no. 14, pp. 1884-1890, 2003.
[4] T. S. Hannon, G. Rao, and S. A. Arslanian, "Childhood obesity and type 2 diabetes mellitus," Pediatrics, vol. 116, no. 2, pp. 473-480, 2005.

[5] F. Bacha, R. Saad, N. Gungor, and S. A. Arslanian, "Are obesityrelated metabolic risk factors modulated by the degree of insulin resistance in adolescents?" Diabetes Care, vol. 29, no. 7, pp. 1599-1604, 2006.

[6] S. B. Racette, E. M. Evans, E. P. Weiss, J. M. Hagberg, and J. O. Holloszy, "Abdominal adiposity is a stronger predictor of insulin resistance than fitness among 50-95 year olds," Diabetes Care, vol. 29, no. 3, pp. 673-678, 2006.

[7] D. B. Allen, B. A. Nemeth, R. R. Clark, S. E. Peterson, J. Eickhoff, and A. L. Carrel, "Fitness is a stronger predictor of fasting insulin levels than fatness in overweight male middleschool children," Journal of Pediatrics, vol. 150, no. 4, pp. 383387, 2007.

[8] S. A. Arngrímsson, T. Sveinsson, I. Gunnarsdóttir, G. I. Pálsson, E. Jóhannsson, and I. Thorsdottir, "The relation of fatness to insulin is independent of fitness in 9- but not 15-yrolds," Medicine and Science in Sports and Exercise, vol. 40, no. 1, pp. 43-49, 2008.

[9] G. D. C. Ball, G. Q. Shaibi, M. L. Cruz, M. P. Watkins, M. J. Weigensberg, and M. I. Goran, "Insulin sensitivity, cardiorespiratory fitness, and physical activity in overweight Hispanic youth," Obesity Research, vol. 12, no. 1, pp. 77-85, 2004.

[10] J. C. Eisenmann, K. D. DuBose, and J. E. Donnelly, "Fatness, fitness, and insulin sensitivity among 7- to 9-year-old children," Obesity, vol. 15, no. 8, pp. 2135-2144, 2007.

[11] S. J. Lee, F. Bacha, N. Gungor, and S. A. Arslanian, "Cardiorespiratory fitness in youth: relationship to insulin sensitivity and $\beta$-cell function," Obesity, vol. 14, no. 9, pp. 1579-1585, 2006.

[12] M. Krekoukia, G. P. Nassis, G. Psarra, K. Skenderi, G. P. Chrousos, and L. S. Sidossis, "Elevated total and central adiposity and low physical activity are associated with insulin resistance in children," Metabolism, vol. 56, no. 2, pp. 206-213, 2007.

[13] J. Z. Kasa-Vubu, C. C. Lee, A. Rosenthal, K. Singer, and J. B. Halter, "Cardiovascular fitness and exercise as determinants of insulin resistance in postpubertal adolescent females," Journal of Clinical Endocrinology and Metabolism, vol. 90, no. 2, pp. 849-854, 2005.

[14] G. Imperatore, Y. J. Cheng, D. E. Williams, J. Fulton, and E. W. Gregg, "Physical activity, cardiovascular fitness, and insulin sensitivity among U.S. adolescents: the National Health and Nutrition Examination Survey, 1999-2002," Diabetes Care, vol. 29, no. 7, pp. 1567-1572, 2006.

[15] J. M. Lee, M. J. Okumura, M. M. Davis, W. H. Herman, and J. G. Gurney, "Prevalence and determinants of insulin resistance among U.S. adolescents: a population-based study," Diabetes Care, vol. 29, no. 11, pp. 2427-2432, 2006.

[16] N. S. Rizzo, J. R. Ruiz, A. Hurtig-Wennlöf, F. B. Ortega, and M. Sjöström, "Relationship of physical activity, fitness, and fatness with clustered metabolilc risk in children and adolescents: the European youth heart study," Journal of Pediatrics, vol. 150, no. 4, pp. 388-394, 2007.

[17] R. R. Pate, C.-Y. Wang, M. Dowda, S. W. Farrell, and J. R. O'Neill, "Cardiorespiratory fitness levels among US youth 12 to 19 years of age: findings from the 1999-2002 national health and nutrition examination survey," Archives of Pediatrics and Adolescent Medicine, vol. 160, no. 10, pp. 1005-1012, 2006.

[18] Centers for Disease Control and Prevention, "NHANES 19992000 public data release file documentation," December 2007, http://www.cdc.gov/nchs/nhanes/nhanes99_00.htm. 
[19] Centers for Disease Control and Prevention, "NHANES 20012002 public data release file documentation," December 2007, http://www.cdc.gov/nchs/nhanes.htm.

[20] Centers for Disease Control and Prevention; National Center for Health Statistics: National Health and Nutrition Examination, "Anthropometry Procedures Manual," US Department of Health and Human Services, Centers for Disease Control and Prevention, Hyattsville, Md, USA, August 2009, http://www.cdc.gov/nchs/data/nhanes/nhanes_03_04/BM.pdf.

[21] R. J. Kuczmarski, C. L. Ogden, L. M. Grummer-Strawn, et al., "CDC growth charts: United States," Advance Data, no. 314, pp. 1-27, 2000.

[22] B. Balkau, J. E. Deanfield, J.-P. Després, et al., "International day for the evaluation of abdominal obesity (IDEA): a study of waist circumference, cardiovascular disease, and diabetes mellitus in 168000 primary care patients in 63 countries," Circulation, vol. 116, no. 17, pp. 1942-1951, 2007.

[23] H. J. Schneider, H. Glaesmer, J. Klotsche, et al., "Accuracy of anthropometric indicators of obesity to predict cardiovascular risk," Journal of Clinical Endocrinology and Metabolism, vol. 92, no. 2, pp. 589-594, 2007.

[24] S. M. Camhi, J. Kuo, and D. R. Young, "Identifying adolescent metabolic syndrome using body mass index and waist circumference," Preventing Chronic Disease, vol. 5, no. 4, article A115, pp. 1-9, 2008.

[25] Centers for Disease Control and Prevention; National Center for Health Statistics, "National Health and Nutrition Examination Cardiovascular Fitness Procedures Manual," US Department of Health and Human Services, Centers for Disease Control and Prevention, Hyattsville, Md, USA, 2004.

[26] Centers for Disease Control and Prevention, "National Center for Health Statistics: National Health and Nutrition Examination Laboratory Manual," US Department of Health and Human Services, Centers for Disease Control and Prevention, Hyattsville, Md, USA, 2004.

[27] M. Keskin, S. Kurtoglu, M. Kendirci, M. E. Atabek, and C. Yazici, "Homeostasis model assessment is more reliable than the fasting glucose/insulin ratio and quantitative insulin sensitivity check index for assessing insulin resistance among obese children and adolescents," Pediatrics, vol. 115, no. 4, pp. e500-e503, 2005.

[28] Y. Manios, G. Moschonis, G. Kourlaba, et al., "Prevalence and independent predictors of insulin resistance in children from Crete, Greece: the Children Study," Diabetic Medicine, vol. 25, no. 1, pp. 65-72, 2008.

[29] J. R. Ruiz, N. S. Rizzo, F. B. Ortega, H. M. Loit, T. Veidebaum, and M. Sjöström, "Markers of insulin resistance are associated with fatness and fitness in school-aged children: the European Youth Heart Study," Diabetologia, vol. 50, no. 7, pp. 14011408, 2007.

[30] G. Q. Shaibi, M. L. Cruz, G. D. C. Ball, et al., "Effects of resistance training on insulin sensitivity in overweight Latino adolescent males," Medicine and Science in Sports and Exercise, vol. 38, no. 7, pp. 1208-1215, 2006.

[31] R. P. Troiano, D. Berrigan, K. W. Dodd, L. C. Mâsse, T. Tilert, and M. McDowell, "Physical activity in the United States measured by accelerometer," Medicine and Science in Sports and Exercise, vol. 40, no. 1, pp. 181-188, 2008.

[32] O. Saygin, E. Zorba, K. Karacabey, and S. Mengutay, "Gender and maturation differences in health-related physical fitness and physical activity in Turkish children," Pakistan Journal of Biological Sciences, vol. 10, no. 12, pp. 1963-1969, 2007.
[33] G. S. Krahenbuhl, J. S. Skinner, and W. M. Kohrt, "Developmental aspects of maximal aerobic power in children," Exercise and Sport Sciences Reviews, vol. 13, pp. 503-538, 1985.

[34] W. S. Cutfield, C. A. Jefferies, W. E. Jackson, E. M. Robinson, and P. L. Hofman, "Evaluation of HOMA and QUICKI as measures of insulin sensitivity in prepubertal children," Pediatric Diabetes, vol. 4, no. 3, pp. 119-125, 2003.

[35] G. Guzzaloni, G. Grugni, G. Mazzilli, D. Moro, and F. Morabito, "Comparison between $\beta$-cell function and insulin resistance indexes in prepubertal and pubertal obese children," Metabolism, vol. 51, no. 8, pp. 1011-1016, 2002. 




The Scientific World Journal
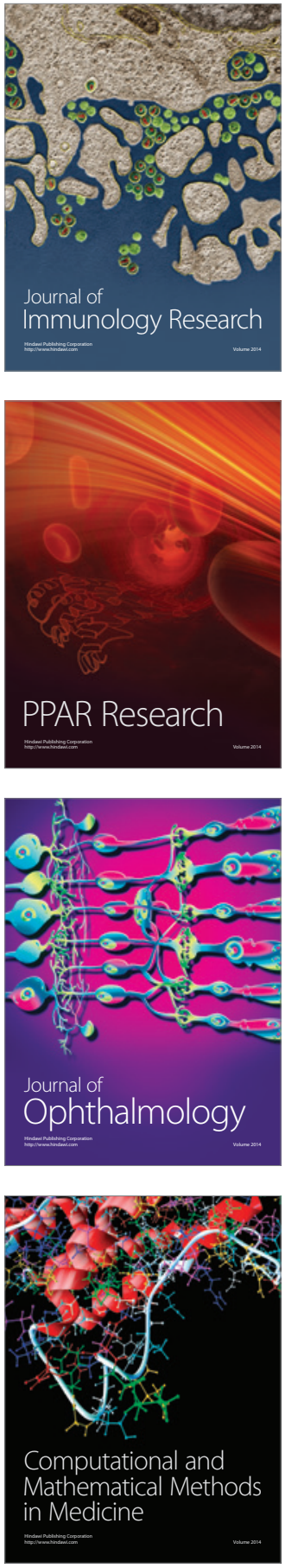

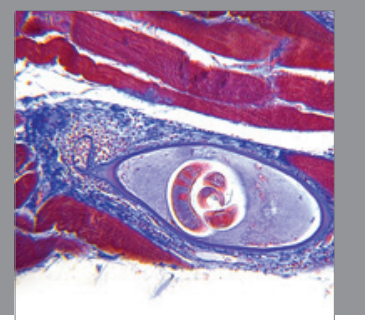

Gastroenterology

Research and Practice
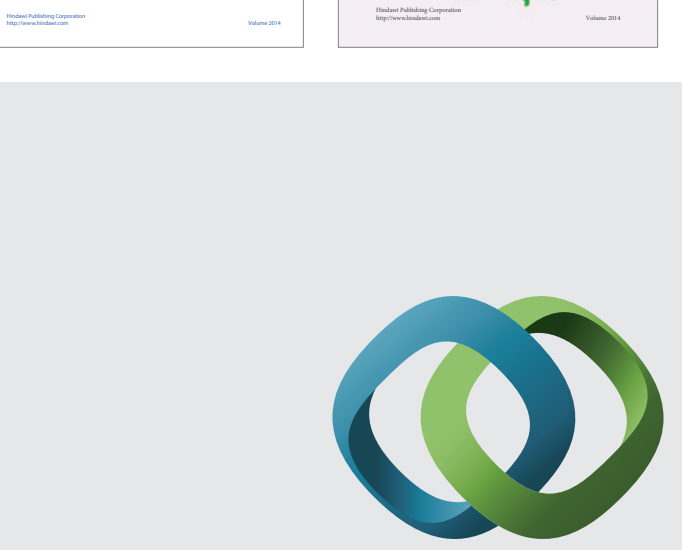

\section{Hindawi}

Submit your manuscripts at

http://www.hindawi.com
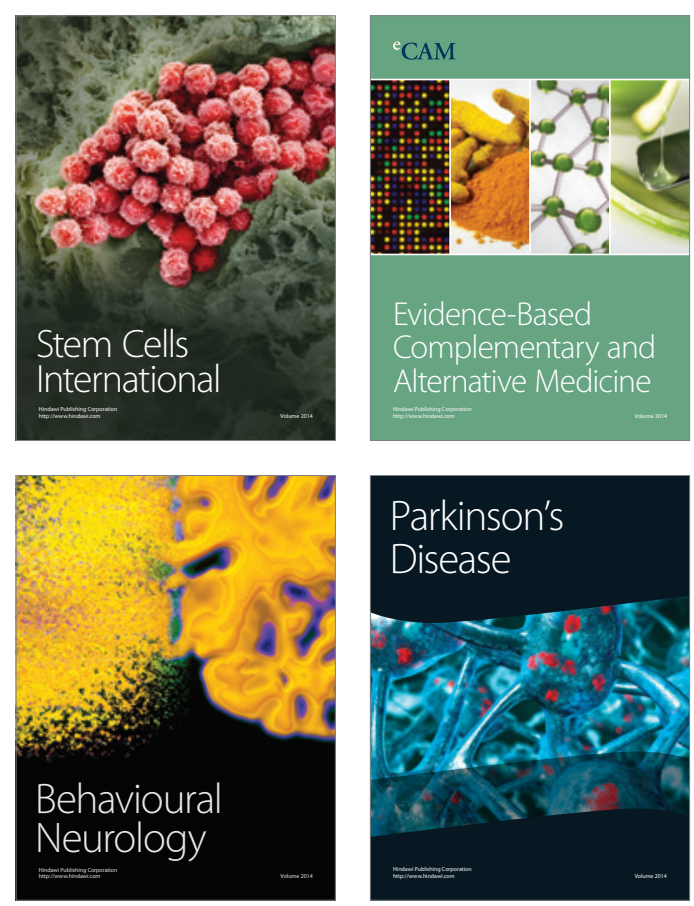

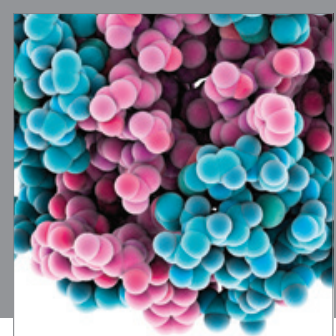

Journal of
Diabetes Research

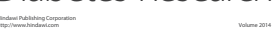

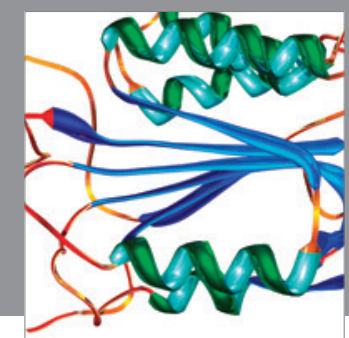

Disease Markers
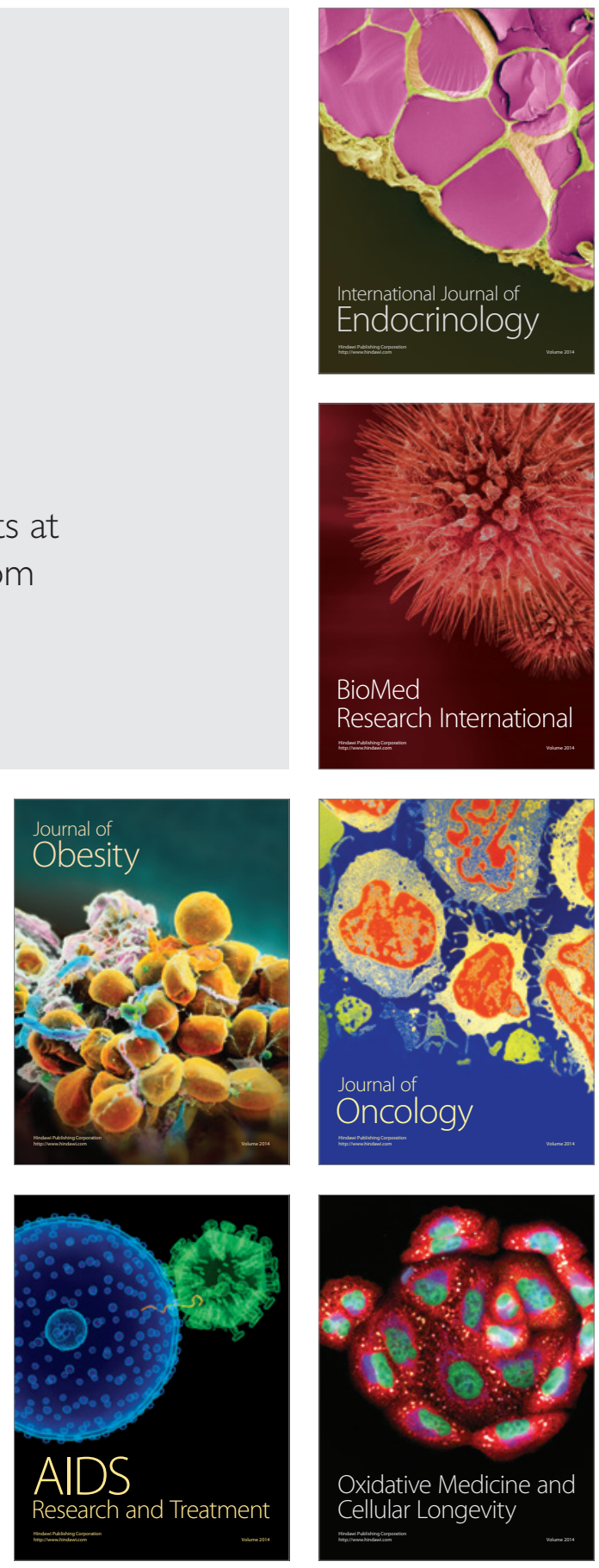